\title{
Disseminated intravascular coagulation following administration of sunitinib
}

\author{
ANAËLLE OLIVO ${ }^{1}$, NICOLAS NOËL ${ }^{2-5}$, BENJAMIN BESSE ${ }^{3,6}$, \\ ANNE-MARIE TABURET ${ }^{1-5}$ and OLIVIER LAMBOTTE TE $^{2,3,5}$
}

\begin{abstract}
${ }^{1}$ Department of Clinical Pharmacy and ${ }^{2}$ Internal Medicine and Clinical Immunology Department, Bicêtre Hospital, AP-HP, F-94270 Kremlin Bicêtre; ${ }^{3}$ Université Paris Sud, Kremlin Bicêtre, F-94270 Kremlin Bicêtre; ${ }^{4}$ Division of Immunovirology, IDMIT, F-92265 Fontenay-aux-Roses; ${ }^{5}$ Center for Immunology of Viral Infections and Autoimmune Diseases, F-92290 Chatenay-Malabry; ${ }^{6}$ Institut Gustave Roussy, F-94800 Villejuif, France
\end{abstract}

Received September 8, 2015; Accepted January 20, 2016

DOI: $10.3892 / \mathrm{mco} .2016 .896$

\begin{abstract}
Sunitinib is an increasingly used, orally administered targeted therapy, approved by the European Medicines Agency for the treatment of various types of cancer, including gastrointestinal stromal tumor unresectable or metastatic disease, following disease progression or intolerance to imatinib, and advanced or metastatic renal cell carcinoma, progressive well-differentiated pancreatic neuroendocrine tumors in patients with unresectable, locally advanced or metastatic disease. Sunitinib inhibits several tyrosine kinases, including the vascular endothelial growth factor receptor and the platelet-derived growth factor receptor. Tyrosine kinases inhibitor therapies are generally well-tolerated; nonetheless, they are not void of side effects. The majority of patients reported are grade 1 or 2 , and include common and unspecific adverse events, including fatigue, gastrointestinal disorders, skin discoloration, altered taste, cough and dyspnea. Grade 3 or 4 adverse events, including bleeding and hemorrhage, are less frequent. The present study presented the first case of disseminated intravascular coagulation associated with the administration of sunitinib, shortly following the increase of sunitinib dosage.
\end{abstract}

\section{Introduction}

Sunitinib is a tyrosine kinase inhibitor (Sutent ${ }^{\circledR}$ Capsules; Pfizer, Kent, UK), approved by the European Medicines Agency for the treatment of unresectable or metastatic gastrointestinal stromal tumors and renal cell carcinoma.

Correspondence to: Professor Olivier Lambotte, Internal Medicine and Clinical Immunology Department, Bicêtre Hospital, AP-HP, 78 Rue du Général Leclerc, F-94270 Kremlin Bicêtre, France

E-mail: olivier.lambotte@aphp.fr

Key words: sunitinib, disseminated intravascular coagulation, hematological toxicity, metastatic carcinoma, pharmacovigilance
Sunitinib inhibits several tyrosine kinases, including vascular endothelial growth factor receptor (VEGFR) and platelet-derived growth factor receptor (PDGFR). Simultaneous inhibition of PDGFR and VEGFR has a crucial role in reducing tumor vascularization and tumor cell proliferation (1). The most common adverse events associated with sunitinib are asthenia and gastrointestinal toxicity. Specific adverse drug reactions include skin discoloration, as well as bleeding and proteinuria, due to the inhibition of angiogenesis (2). Inhibition of VEGF impairs tissue repair and renewal of endothelial cells, and may lead to an elevated risk of bleeding. The overall incidence of hemorrhage (all grades) is $19.3 \%$. However, severe hemorrhage is rare, with an incidence of $3 \%$ for grade 3-5 events (3).

Additionally, sunitinib can induce myelosuppression (4). According to the summary of product characteristics, thrombocytopenia occurs in $8.9 \%$ of patients.

The present study described a case of disseminated intravascular coagulation, associated with the administration of sunitinib. To the best of our knowledge, this is the first report of this adverse event.

\section{Case report}

A 34-year-old male patient with Type I diabetes was undergoing treatment for invasive thymoma (diagnosed in 2012) with pulmonary, bone, liver and pleural metastases. For $>1$ year, the patient had been suffering from fever, a chronic inflammatory syndrome and anemia, requiring regular red blood cell transfusions.

The patient initially received etoposide-cisplatin, followed by cisplatin-doxorubicin-cyclophosphamide. This treatment produced a partial response, however, the patient developed chronic kidney disease as a result of cisplatin administration and diabetes. The disease recurred in February 2014. A further course of etoposide-carboplatin was initiated, however, did not prevent disease progression within the liver. Biopsy of a liver nodule revealed metastases of thymic carcinoma. Treatment with sunitinib was initiated in July 2014 , termed as day 0. A low dose (25 mg/daily) was used since the patient had an Eastern Cooperative 


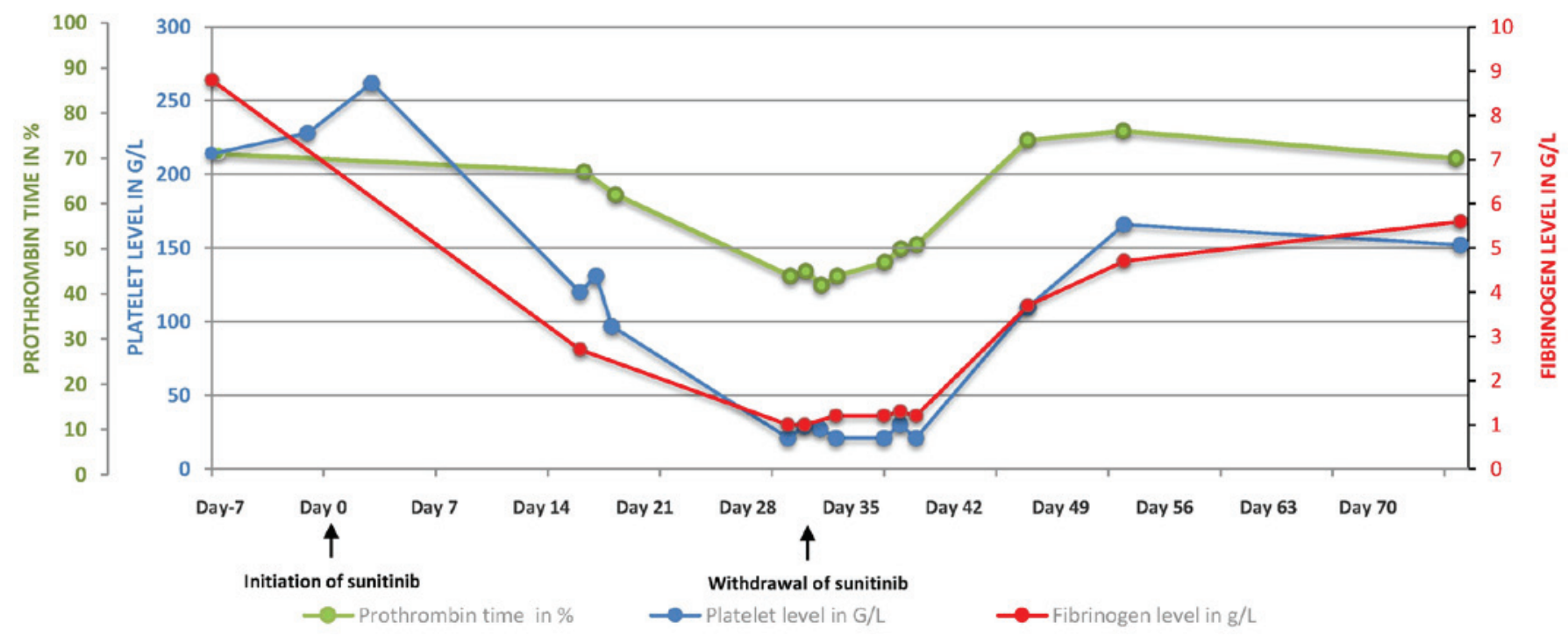

Figure 1. Evolution of fibrinogen and platelet levels and prothrombin time following the initiation of sunitinib.

Oncology Group performance status of 3. The patient also received amlodipine, esomeprazole, insulin and furosemide. Upon initiation of sunitinib, the results of the complete blood count and coagulation tests were normal: The blood levels of hemoglobin $(9.2 \mathrm{~g} / \mathrm{dl})$, creatinine $(189 \mu \mathrm{mol} / \mathrm{l})$ and fibrinogen $(8 \mathrm{~g} / \mathrm{l})$. Two weeks later, the daily dose of sunitinib was increased to $37.5 \mathrm{mg}$. A computed tomography scan on day 21 showed that the disease was stable. The patient soon complained of edema of the lower limbs and the platelet count fell to $100 \mathrm{G} / 1$ after three weeks of treatment (Fig. 1).

On day 28, the patient was admitted to the Emergency Department after noticing spots of blood on his underwear. No evidence of gastrointestinal or urinary hemorrhage was observed. The body temperature and blood pressure were normal. The patient complained about worsening of the white, soft, painless edema of the lower limbs over the previous month. Neither proteinuria nor hypertension was observed, thus ruling out nephrotic syndrome. A hematological assessment on day 29 revealed bicytopenia, with a hemoglobin level of $6.1 \mathrm{~g} / \mathrm{dl}$, a platelet count of $21 \mathrm{G} / 1$ and a white blood cell count of $4.9 \mathrm{G} / 1$ (Fig. 1). The levels of haptoglobin and unconjugated bilirubin were normal, thus ruling out hemolysis. No schistocytosis was diagnosed. The present study diagnosed disseminated intravascular coagulation (DIC), on the basis of thrombocytopenia, a low prothrombin time (PT; $45 \%)$ and low levels of factors II (65\%), V (44\%), VII (57\%) and $\mathrm{X}(121 \%)$, and fibrinogen $(1.3 \mathrm{~g} / \mathrm{l})$. The patient received four red blood cell units and two platelet units. Sunitinib was withdrawn. The hematological situation worsened, with an increased requirement for transfusion and the development of purpuric lesions on the left arm, purpuric and ecchymotic lesions in the abdomen, and bruising at the injection site. The levels of coagulation factors fell to $<50 \%$, with the exception of factor II, at 57\%), with soluble complexes $>150 \mu \mathrm{g} / \mathrm{ml}$ and fibrin degradation products $>40 \mu \mathrm{g} / \mathrm{ml}$. The nadirs of the platelet count and the hemoglobin level were $21 \mathrm{G} / 1$ and $5.7 \mathrm{~g} / \mathrm{dl}$, respectively.

The DIC observed may potentially have been associated with tumor progression, with possible bone marrow involve- ment, infectious disease or an iatrogenic effect of sunitinib. A bone marrow aspirate revealed a moderately rich marrow and a normal megakaryocyte profile, with no malignant cells and no hemophagocytosis. Bacterial cultures were negative. The patient received a further four red blood cell units and two units of platelet concentrate. The withdrawal of sunitinib and the initiation of hematological support were followed by a slow improvement in the clinical signs of hemorrhagic syndrome, which resolved 10 days later. The platelet counts, PT and fibrinogen levels normalized within 15 days. Once the DIC had resolved, a sixth course of treatment with paclitaxel was initiated 6 weeks after the final administration of sunitinib. Although no recurrence of DIC was reported, the patient became neutropenic and succumbed to septic shock. The present study, therefore, suspected sunitinib to be causally associated with the severe hematological toxicity associated with DIC.

\section{Discussion}

To the best of our knowledge, this is the first report of DIC with a probable causal association with sunitinib.

The initial toxic effects of sunitinib (lower limb edema) appeared early in the course of treatment and worsened following an increase in the dose. The edema may have been due to the inhibition of PDGF, which is known to be involved in fluid homeostasis (5), or to hypoalbuminemia. Disseminated intravascular coagulation developed within 4 weeks of therapy. The usual causes of DIC, bacterial sepsis and bone marrow invasion by malignant cells, were absent (6). Sunitinib was the only drug prescribed after July 2014. Therefore, after eliminating other likely causes, it was hypothesized that sunitinib had an iatrogenic effect, as evidenced by the reduction in clinical and biological symptoms, including complete normalization of coagulation parameters in the context of metastatic carcinoma, following treatment discontinuation. Therefore, regular monitoring of the complete blood count and coagulation parameters is advisable in patients treated with sunitinib, according to the summary of product characteristics (7). 


\section{References}

1. Aparicio-Gallego G, Blanco M, Figueroa A, García-Campelo R, Valladares-Ayerbes M, Grande-Pulido E and Antón-Aparicio L: New insights into molecular mechanisms of sunitinib-associated side effects. Mol Cancer Ther 10: 2215-2223, 2011.

2. Kollmannsberger C, Soulieres D, Wong R, Scalera A, Gaspo R and Bjarnason G: Sunitinib therapy for metastatic renal cell carcinoma: recommendations for management of side effects. Can Urol Assoc J 1: S41-S54, 2007.

3. Je Y, Schutz FA and Choueiri TK: Risk of bleeding with vascular endothelial growth factor receptor tyrosine-kinase inhibitors sunitinib and sorafenib: A systematic review and meta-analysis of clinical trials. Lancet Oncol 10: 967-974, 2009.
4. Kumar R, Crouthamel MC, Rominger DH, Gontarek RR, Tummino PJ, Levin RA and King AG: Myelosuppression and kinase selectivity of multikinase angiogenesis inhibitors. Br J Cancer 101: 1717-1723, 2009.

5. Giles FJ, O'Dwyer M and Swords R: Class effects of tyrosine kinase inhibitors in the treatment of chronic myeloid leukemia. Leukemia 23: 1698-1707, 2009.

6. Levy M: Disseminated intravascular coagulation: What's New? Crit Care Clin 21: 449-467, 2005.

7. Valle J W, Faivre S, Hubner R A, Grande E and Raymond E: Practical management of sunitinib toxicities in the treatment of pancreatic neuroendocrine tumors. Cancer Treat Rev 40: 12301238,2014 